\title{
Understanding Power Spectrum Density Transmissibility
}

\author{
Yu Zhang, Tong Zhu, and Jing Zhou \\ State Key Lab of Coastal and Offshore Engineering, Dalian University of Technology, Dalian 116024, China \\ Correspondence should be addressed to Yu Zhang; zyuyunhui@yeah.net
}

Received 3 August 2015; Revised 6 November 2015; Accepted 15 November 2015

Academic Editor: Salvatore Russo

Copyright (C) 2016 Yu Zhang et al. This is an open access article distributed under the Creative Commons Attribution License, which permits unrestricted use, distribution, and reproduction in any medium, provided the original work is properly cited.

\begin{abstract}
Power spectrum density transmissibility (PSDT) is a type of complex frequency domain function proposed recently. It describes the relation between cross-spectra of system outputs. Since PSDTs with same local-reference degree of freedom (DOF) combination but with different transferring output DOFs cross each other at the system's poles under certain load condition, the functions have been used as the primary data in operational modal analysis (OMA) to extract modal parameters, and such technique is named as PSDT-based OMA (PSDTOMA). Because PSDT is a concept that appears recently, researches on which, especially, in-depth discussions aim at the essence and properties of PSDT are very rare. For appropriate application of PSDTOMA, it is necessary to perform further study on such problem obviously. In this paper, two paths to get PSDT, which, respectively, are referred to as PSDT estimator and PSDT syntheticism, are given firstly; some properties about PSDT are explored based on the PSDT syntheticism, and the relation between PSDT and single reference transmissibility function (STF) is analyzed. Finally, the above conclusions are verified with numerical values and experimental data.
\end{abstract}

\section{Introduction}

Similar to more common-used transfer functions, transmissibility is a type of (complex) frequency domain functions, which describe the relation between physical quantities of the same type at different locations in a dynamical system. According to the type of physical quantities described, the transmissibility about system dynamic characteristics can be divided into force transmissibility and motion transmissibility.

Power spectrum density transmissibility (PSDT), which this paper studies, was first proposed by Yan and Ren [1] in 2012 as the third kind of motion transmissibility following the traditional single reference transmissibility function (STF) [2-4] and multi-/poly-reference transmissibility function (MTF/PTF) [5-7], and it is defined as the ratio of crossspectra between responses at different locations in a system. Similar to STF, PSDT is a scalar (complex) frequency domain function as well, representing the relation between responses of local degree of freedom (DOF) and reference DOF. Because PSDT introduces the concept of transferring output DOF additionally and the PSDT, depending on different transferring output DOFs, intersects at the system poles under certain loading condition $[1,8]$, in theory, the STF, depending on different loading conditions, can be replaced with the PSDT, depending on different transferring output DOFs, which is used as the primary data of operational modal analysis (OMA) to lower identification method's demand on the number of loading conditions. This OMA process is called PSDT-based OMA (PSDTOMA). Same as STFbased OMA (sTOMA), PSDTOMA has the characteristic that identification results are unaffected by harmonic component in excitation, compared to the traditional OMA methods.

Because PSDT was proposed recently, it has not been studied thoroughly and deeply. To the authors' knowledge, all the current PSDT-related studies focus on the application layer $[1,8]$, and no discussion on its essence and property has been made. In this paper, two paths to get PSDT, which, respectively, are referred to as PSDT estimator and PSDT syntheticism, are given firstly; some properties about PSDT are explored based on the PSDT syntheticism, and the relation between PSDT and single reference transmissibility function (STF) which is similar to PSDT is analyzed. Finally, the above conclusions are verified with numerical values and experimental data. 


\section{Power Spectrum Density Transmissibility}

Under a certain load condition, PSDT is defined as the ratio of cross-spectral densities between response at DOF $p$ and responses at DOFs $i, j$, respectively $[1,8]$. It is expressed as

$$
\operatorname{psdt}_{i j}^{p}(\omega)=\frac{S_{y_{i} y_{p}}(\omega)}{S_{y_{j} y_{p}}(\omega)}
$$

where $S_{y_{i} y_{p}}(\omega)$ and $S_{y_{j} y_{p}}(\omega)$ are cross-spectral densities between responses at DOFs $i, p$ and responses at DOFs $j, p$, respectively; DOFs $i, j$, and $p$ are referred to as local DOF, reference DOF, and transferring output DOF, respectively.

In the frequency domain, the relation between $N_{i}$ inputs and $N_{o}$ outputs of a linear time invariant (LTI) system can be expressed as

$$
\left[S_{y y}(\omega)\right]=[H(\omega)]\left[S_{f f}(\omega)\right][H(\omega)]^{H},
$$

where $\left[S_{y y}(\omega)\right]$ is $N_{o} \times N_{o}$ output power spectrum matrix; $\left[S_{f f}(\omega)\right]$ is $N_{i} \times N_{i}$ input power spectrum matrix; $[H(\omega)]$ is $N_{o} \times N_{i}$ transfer function matrix; $[H(\omega)]^{H}$ denote the complex conjugate and transpose of $[H(\omega)]$. According to (2), the cross-spectral density between response at two DOFs, such as $i$ and $p$, which also is the entry in the $i$ th row and $p$ th column of $\left[S_{y y}(\omega)\right]$, can be expressed as

$$
S_{y_{i} y_{p}}(\omega)=\left\langle H_{i}(\omega)\right\rangle\left[S_{f f}(\omega)\right]\left\langle H_{p}(\omega)\right\rangle^{H},
$$

where $\left\langle H_{i}(\omega)\right\rangle$ and $\left\langle H_{p}(\omega)\right\rangle$ are row vectors consisting of entries in the $i$ th and $p$ th row of $[H(\omega)]$, respectively. Combining (1) and (3), we get another expression of PSDT:

$$
\operatorname{psdt}_{i j}^{p}(\omega)=\frac{\left\langle H_{i}(\omega)\right\rangle\left[S_{f f}(\omega)\right]\left\langle H_{p}(\omega)\right\rangle^{H}}{\left\langle H_{j}(\omega)\right\rangle\left[S_{f f}(\omega)\right]\left\langle H_{p}(\omega)\right\rangle^{H}} .
$$

Equations (1) and (4) are two opposite paths to get the value of PSDT. Equation (1) is referred to as PSDT estimator, which is used for getting PSDT estimation from outputs, while (4) is referred to as PSDT syntheticism, which is used for getting PSDT synthesis from inputs and transfer function. The relation among PSDT syntheticism, PSDT estimator, PSDT synthesis, and PSDT estimation is shown in Figure 1. In theory, PSDT estimation is a consistent approach of PSDT synthesis, and this makes PSDT synthesis be regarded as an evaluation criterion for PSDT estimation.

Actually, the result obtained by putting random excitations into (4) directly is an approximation of PSDT. It is because there exists interference caused by spectral leakage. In some cases, however, (4) can be further simplified to get the exact value of PSDT. In theory [9], the energy of the stationary random process, $x$, is distributed uniformly in the frequency domain, so the amplitude of its autospectrum $S_{x x}$ is unrelated to (circle) frequent $\omega$ and proportional to variance of the process $\sigma_{x}^{2}$, and it can be expressed as

$$
S_{x x}(\omega)=\alpha^{2} \cdot \sigma_{x}^{2}
$$

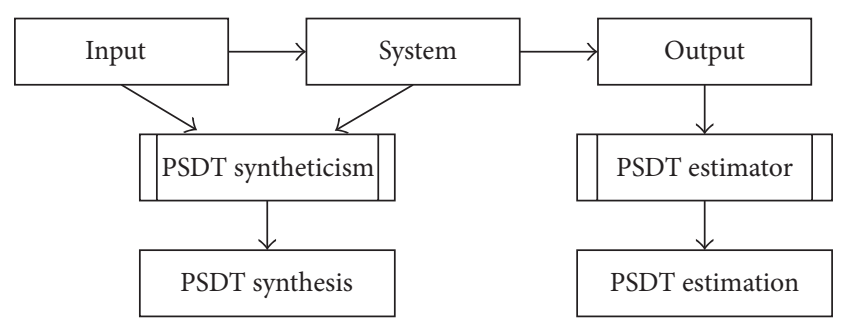

FIGURE 1: PSDT syntheticism, PSDT estimator, PSDT synthesis, and PSDT estimation.
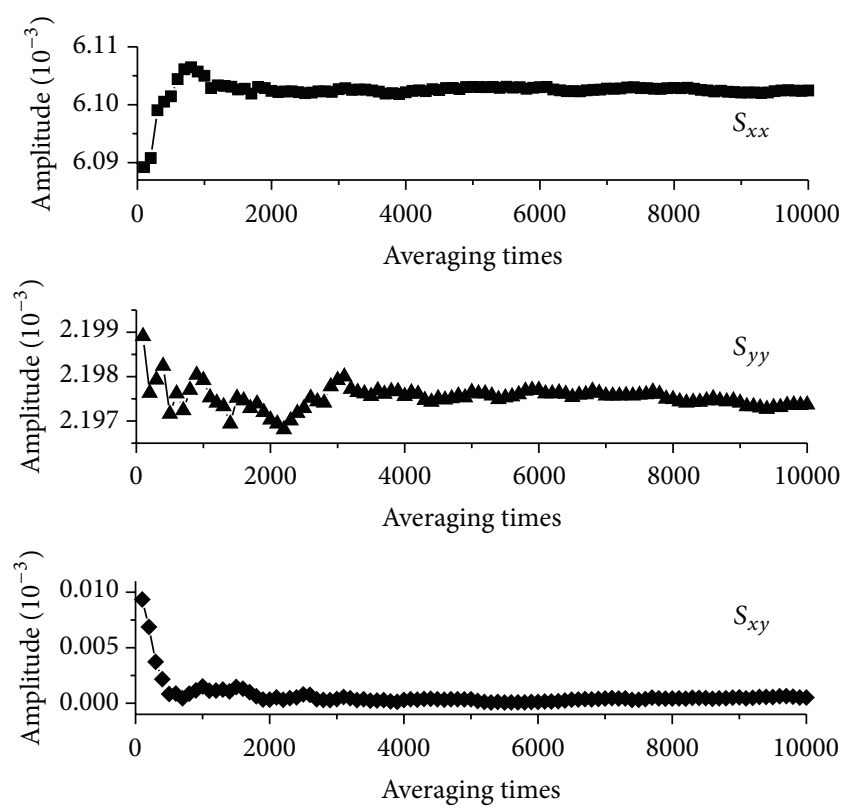

FIgURE 2: Amplitudes of random process's PSD $\left(\sigma_{x}=5, \sigma_{y}=3\right)$.

where $\alpha$ is a real nonzero proportion factor, related to the sampling length. Therefore, if two stationary random processes, $x$ and $y$, are fully correlative, the amplitude of their cross-spectrum $S_{x y}$ is proportional to covariance of these two processes $\sigma_{x} \sigma_{y}$, and it can be expressed as

$$
\left|S_{x y}(\omega)\right|=\alpha^{2} \cdot \sigma_{x} \sigma_{y} .
$$

Nevertheless, if $x$ and $y$ are mutually uncorrelated zero-mean random processes, the amplitude of cross-spectrum $S_{x y}$ will approach zero after averaging many times; that is, $\left|S_{x y}(\omega)\right|=$ 0 , as shown in Figure 2.

According to (5) and (6), the amplitude of matrix $\left[S_{f f}(\omega)\right]$ can be uniformly expressed as

$$
\left[\left|S_{f f}(\omega)\right|\right]=\alpha^{2} \cdot[\Gamma]
$$


In the case that the system's excitations are correlated completely, the expression of matrix $[\Gamma]$ is as follows:

$$
[\Gamma]=\left[\begin{array}{cccc}
\sigma_{1}^{2} & \sigma_{1} \sigma_{2} & \cdots & \sigma_{1} \sigma_{N_{i}} \\
\sigma_{2} \sigma_{1} & \sigma_{2}^{2} & \cdots & \sigma_{2} \sigma_{N_{i}} \\
\vdots & \vdots & \ddots & \vdots \\
\sigma_{N_{i}} \sigma_{1} & \sigma_{N_{i}} \sigma_{2} & \cdots & \sigma_{N_{i}}^{2}
\end{array}\right],
$$

where $\sigma_{1}, \sigma_{2}, \ldots, \sigma_{N_{i}}$ are the standard deviations of $N_{i}$ random excitations. In the case that all the $N_{i}$ excitations are fully independent, the value of $[\Gamma]$ is the diagonal matrix as follows:

$$
[\Gamma]=\left[\begin{array}{cccc}
\sigma_{1}^{2} & 0 & \cdots & 0 \\
0 & \sigma_{2}^{2} & \cdots & 0 \\
\vdots & \vdots & \ddots & \vdots \\
0 & 0 & \cdots & \sigma_{N_{i}}^{2}
\end{array}\right]
$$

Finally, if excitations are partially correlative, the value of $[\Gamma]$ can be obtained by appropriately choosing off-diagonal entries. Based on the above discussion, (4) can be rewritten as

$$
\left|\operatorname{psdt}_{i j}^{p}(\omega)\right|=\left|\frac{\left\langle H_{i}(\omega)\right\rangle[\Gamma]\left\langle H_{p}(\omega)\right\rangle^{H}}{\left\langle H_{j}(\omega)\right\rangle[\Gamma]\left\langle H_{p}(\omega)\right\rangle^{H}}\right|
$$

Thus, PSDT's amplitude can be obtained exactly by using (10).

Let $\{\sigma\}$ be the following vector:

$$
\{\sigma\}=\left\langle\begin{array}{llll}
\sigma_{1} & \sigma_{2} & \cdots & \sigma_{N_{i}}
\end{array}\right\rangle^{T} .
$$

$[\Gamma]$ in (8) can be expressed as

$$
[\Gamma]=\{\sigma\}\{\sigma\}^{T} .
$$

By putting (12) into (10), we get

$$
\begin{aligned}
\left|\operatorname{psdt}_{i j}^{p}(\omega)\right| & =\left|\frac{\left\langle H_{i}(\omega)\right\rangle\{\sigma\}\{\sigma\}^{T}\left\langle H_{p}(\omega)\right\rangle^{H}}{\left\langle H_{j}(\omega)\right\rangle\{\sigma\}\{\sigma\}^{T}\left\langle H_{p}(\omega)\right\rangle^{H}}\right| \\
& =\left|\frac{\left\langle H_{i}(\omega)\right\rangle\{\sigma\}}{\left\langle H_{j}(\omega)\right\rangle\{\sigma\}}\right| .
\end{aligned}
$$

Obviously, in the case that the system's excitations are fully independent, the value of PSDT's amplitude is unrelated to the way of selecting transferring output DOF $p$. More particularly, in the case that the system is subject to single concentrated excitation, for instance, the force is acting on DOF $k$; (10) degrades into the following form:

$$
\left|\operatorname{psdt}_{i j}^{p}(\omega)\right|=\left|\frac{H_{i k}(\omega) \sigma_{k} H_{p k}(\omega)}{H_{j k}(\omega) \sigma_{k} H_{p k}(\omega)}\right|=\left|\frac{H_{i k}(\omega)}{H_{j k}(\omega)}\right|
$$

which means that the amplitude of PSDT depends on the location, rather than the strength of excitation.

\section{Power Spectrum Density Transmissibility and Single Reference Transmissibility}

STF is the other kind of scalar motion transmissibility for MDOF system. In frequency domain, STF is defined as the ratio of Fourier transforms of responses at local DOF $i\left(Y_{i}(s)\right)$ and DOF $j\left(Y_{j}(s)\right)[2-4]$; that is,

$$
t_{i j}(\omega)=\frac{Y_{i}(\omega)}{Y_{j}(\omega)}
$$

Besides (2), the relation between inputs and outputs of LTI system can also be expressed as

$$
\{Y(\omega)\}=[H(\omega)]\{F(\omega)\},
$$

where $\{F(s)\}$ is $N_{i} \times 1$ input spectral vector and $\{Y(s)\}$ is $N_{o} \times$ 1 output spectral vector. According to (16), the response at some DOF, such as $i$, can be expressed as

$$
Y_{i}(\omega)=\left\langle H_{i}(\omega)\right\rangle\{F(\omega)\} .
$$

By combining (15) and (17), we can get

$$
t_{i j}(\omega)=\frac{\left\langle H_{i}(\omega)\right\rangle\{F(\omega)\}}{\left\langle H_{j}(\omega)\right\rangle\{F(\omega)\}} .
$$

Similar to (4), (18) can be used to synthesize STF from input information and transfer function. It is important to note that because there exists infinite error introduced by end effects [10], nothing but useless result would be obtained by putting random excitations directly. Hence, (18) can only be used to get STF synthesis in deterministic case.

Consider that the autospectrum of random process $x$, $S_{x x}(\omega)$, can be expressed as

$$
S_{x x}(\omega)=\frac{1}{T} X(\omega) X^{*}(\omega),
$$

where $T$ is the length of time history; $X(\omega)$ is the Fourier transform of $x(t) ; X^{*}(\omega)$ denotes the complex conjugate of $X(\omega)$. By combining (5) and (19), we can get the following equation:

$$
|X(\omega)|=\sqrt{\alpha^{2} T} \cdot \sigma_{x} .
$$

Therefore, the amplitude of input vector $\{F(\omega)\}$ can be expressed as

$$
\{|F(\omega)|\}=\sqrt{\alpha^{2} T}\left\langle\begin{array}{llll}
\sigma_{1} & \sigma_{2} & \cdots & \sigma_{N_{i}}
\end{array}\right\rangle^{T}=\sqrt{\alpha^{2} T}\{\sigma\},
$$

where $\sigma_{1}, \sigma_{2}, \ldots, \sigma_{N_{i}}$ are the standard deviations of $N_{i}$ random excitations, respectively. Putting (21) into (18), we get

$$
\left|t_{i j}(\omega)\right|=\left|\frac{\left\langle H_{i}(\omega)\right\rangle \sqrt{\alpha^{2} T}\{\sigma\}}{\left\langle H_{j}(\omega)\right\rangle \sqrt{\alpha^{2} T}\{\sigma\}}\right|=\left|\frac{\left\langle H_{i}(\omega)\right\rangle\{\sigma\}}{\left\langle H_{j}(\omega)\right\rangle\{\sigma\}}\right| .
$$

Equation (22) can be used to synthesize the amplitude of STF under random excitation, since it is unaffected by end effects and spectral leakage. Again, in the case that the system is 


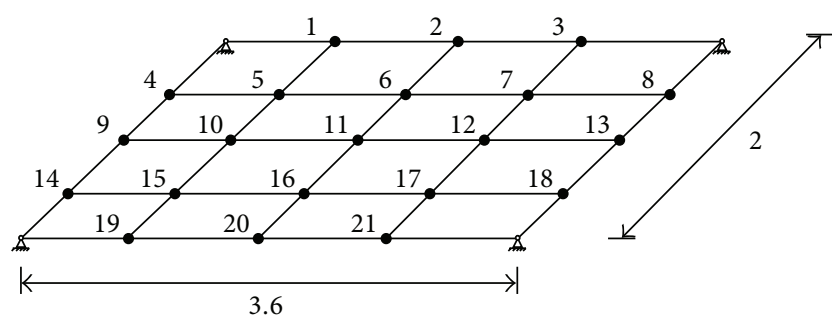

FIGURE 3: Simply supported slab model (size in meters).

subject to single concentrated excitation, (22) degrades into the following form:

$$
\left|t_{i j}(\omega)\right|=\left|\frac{H_{i k}(\omega) \sigma_{k}}{H_{j k}(\omega) \sigma_{k}}\right|=\left|\frac{H_{i k}(\omega)}{H_{j k}(\omega)}\right| .
$$

By comparing (22) with (13), as well as (23) with (14), we get

$$
\left|\operatorname{psdt}_{i j}^{p}(\omega)\right|=\left|t_{i j}(\omega)\right|
$$

Therefore, in the cases that the system is subject to single concentrated excitation and all the inputs of the system are correlated completely, PSDT is exactly identical with the STF with the same local-reference DOF combination.

\section{Numerical Study}

Next, the above conclusions were verified by using the simply supported slab shown in Figure 3. The slab's finite element model (FEM) was established by using ANSYS software. The model, whose size is $3.6 \mathrm{~m} \times 2 \mathrm{~m} \times 0.1 \mathrm{~m}$, was uniformly divided into 720 elements. Its modulus of elasticity is $2 \times$ $10^{11} \mathrm{~N} / \mathrm{m}^{2}$; material density is $7800 \mathrm{~kg} / \mathrm{m}^{3}$; Rayleigh damping coefficients $\alpha$ and $\beta$ are $3.0 \mathrm{~s}^{-1}$ and $1 \times 10^{-4} \mathrm{~s}$, respectively. 21 nodes on the upper surface of the model were selected at equal spacing as the control points used to apply excitations and obtain responses. The positions and numbers of these control points are shown in Figure 3.

13 kinds of random excitation loading conditions, listed in Table 1, were applied to the model successively and the responses at all the 21 control points were extracted. After that, amplitudes of PSDT measurements and syntheses were obtained by using (1) and (10), respectively. For comparison, amplitudes of STFs were synthesized by using (13).

By using the loading conditions in case 1 and case 2, the PSDT syntheses, depending on different transferring output DOFs, are shown in Figure 4. It can be seen that the PSDT syntheses coincide exactly with the corresponding STF synthesis under the two loading conditions, no matter what the transferring output DOF is. Figure 5 shows STF and PSDT syntheses in case 13, from which it can be seen that STF and PSDTs are no longer identical, and the value of PSDT changes with transferring output DOF's changing.

The PSDT measurements, respectively, in single input condition (case 1) and single source condition (case 2), with corresponding STF syntheses under the two loading conditions, are shown in Figure 6. With reference to Figure 4,
TABLE 1: Load conditions for numerical case.

\begin{tabular}{lcc}
\hline Load condition & Input type & Location \\
\hline Case 1 & Single & 5 \\
Case 2 & Full correlative & $1,3,4,6,8,10,12,14,16,18,19,21$ \\
Case 3 & Identical & $1,3,4,6,8,10,12,14,16,18,19$ \\
& Independent & 21 \\
Case 4 & Identical & $1,3,4,6,8,10,12,14,16,18$ \\
& Independent & 19,21 \\
Case 5 & Identical & $1,3,4,6,8,10,12,14,16$ \\
& Independent & $18,19,21$ \\
Case 6 & Identical & $1,3,4,6,8,10,12,14$ \\
& Independent & $16,18,19,21$ \\
Case 7 & Identical & $1,3,4,6,8,10,12$ \\
& Independent & $14,16,18,19,21$ \\
Case 8 & Identical & $1,3,4,6,8,10$ \\
& Independent & $12,14,16,18,19,21$ \\
Case 9 & Identical & $1,3,4,6,8$ \\
& Independent & $10,12,14,16,18,19,21$ \\
Case 10 & Identical & $1,3,4,6$ \\
& Independent & $8,10,12,14,16,18,19,21$ \\
Case 11 & Identical & $1,3,4$ \\
& Independent & $6,8,10,12,14,16,18,19,21$ \\
Case 12 & Identical & 1,3 \\
Case 13 & Independent & $4,6,8,10,12,14,16,18,19,21$ \\
& Independent & $1,3,4,6,8,10,12,14,16,18,19,21$ \\
\hline
\end{tabular}

it can be seen that PSDT measurements coincide exactly with PSDT syntheses under the two loading conditions. The PSDT measurements and PSDT syntheses in case 13 are shown in Figure 7. Despite the uncertain components caused by fewer averaging times (about 100 times), spectral leakage in PSDT measurements, and other factors, it still can be seen clearly that PSDT syntheses and PSDT measurements are identical. Hence the correctness of PSDT syntheticism ((10), (13) and (14)) is proved.

Comparing with loading condition in case 2 , the input applied to point 21 was replaced with a random process uncorrelated with all the other inputs in case 3 . From Figure 8 it can be seen that the PSDT measurements, depending on $p$, were no longer identical with STF synthesis.

Furthermore, the function $\varepsilon(i, j, p)$ is defined to measure the similarity between STF syntheses and PSDT measurements, and it is expressed as

$$
\varepsilon(i, j, p)=\frac{\left(\sum_{k=1}^{N_{f}} \operatorname{psdt}_{i j}^{p}\left(\omega_{k}\right) \cdot t_{i j}\left(\omega_{k}\right)\right)^{2}}{\sum_{k=1}^{N_{f}}\left(\operatorname{psdt}_{i j}^{p}\left(\omega_{k}\right)\right)^{2} \cdot \sum_{k=1}^{N_{f}}\left(t_{i j}\left(\omega_{k}\right)\right)^{2}},
$$

where $\varepsilon$ is within $0-1$; the bigger the value is, the closer the PSDT measurements and STF syntheses are, with "1" indicating the two are identical. In case 2 -case 13 , the relation between $\varepsilon$, depending on different transferring output DOFs, and loading conditions is shown in Figure 9. In the case that excitations are fully correlated, the values of $\varepsilon$ between all the PSDT measurements and STF syntheses were close to 1 , 


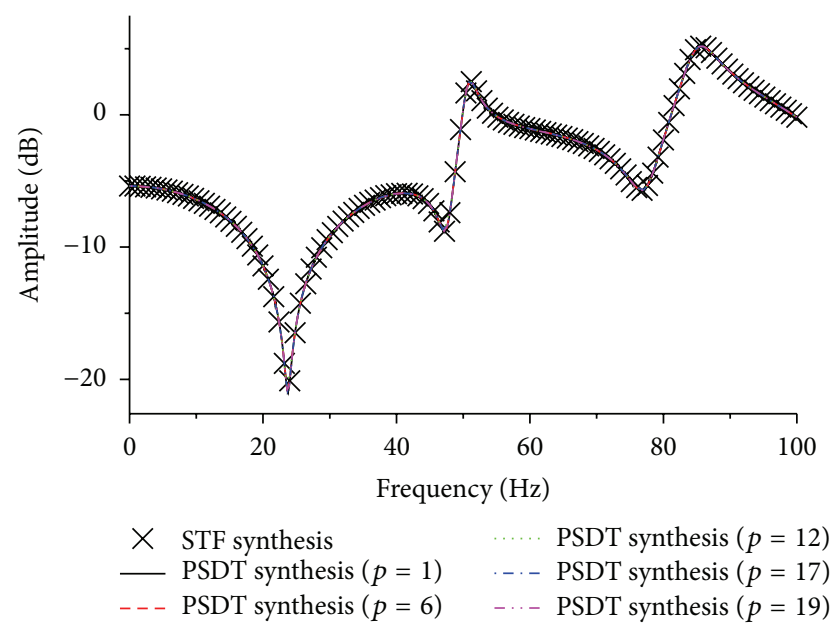

(a)

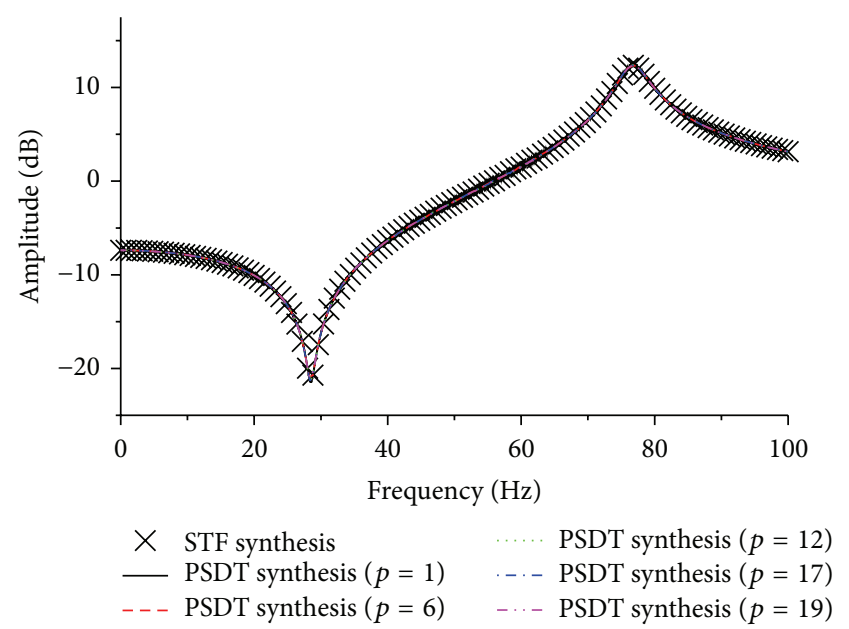

(b)

FIgURE 4: STF and PSDT syntheses with point 4 as local DOF and point 17 as reference DOF in case 1 (a) and case 2 (b).

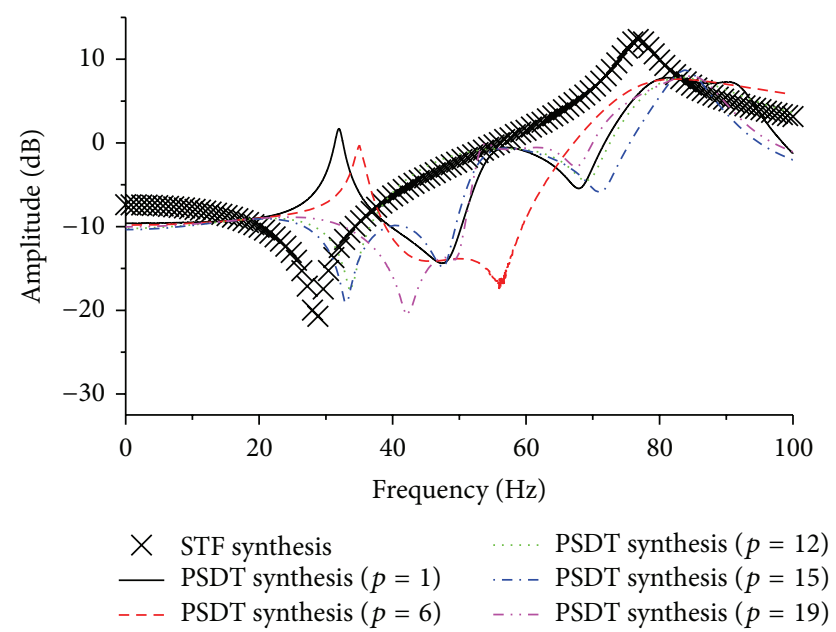

FIGURE 5: STF and PSDT syntheses with point 4 as local DOF and point 17 as reference DOF in case 13.

while, under other loading conditions, no obvious correlation between $\varepsilon$ values and the number of independent excitations was found.

\section{Experimental Study}

Single source excitation is a commonly used mode of excitation, so the above conclusions can be verified easily in practical applications. Figure 10 shows an experimental setup of submarine pipeline vibration-table model. 10 acceleration sensors were installed on the model from south to north, respectively, at the measure points $1-10$, to measure the model's responses in the east-west direction, as shown in Figure 11. When the submarine pipeline is not immersed in water (left graph in Figure 10), the input of the model is totally provided by the vibration-table surface, and thus it can be regarded as a single source excitation system. When the submarine pipeline is immersed in water (right graph in Figure 10), the model is subject to the vibration table, water flow, and waves simultaneously, and thus it can be seen as a random multiple excitation system.

This study takes the loading condition that the model, which was not immersed in water, was only subject to the $0-80 \mathrm{~Hz}$ random east-west excitation provided by the vibration table as case 1 and takes the loading condition that the model, which was immersed in water, was subject to both the excitation in case 1 and the east-west action of water flow and waves as case 2. Figures 12 and 13 show the PSDT measurements obtained based on model responses and, respectively, taking the measure points $2,6,5$, and 9 as local DOFs and reference DOFs. From them it can be seen that, in case 1 , the PSDT obtained by taking the response of any one of the measure points as transferring output was basically identical to each other (Figures 12(a) and 13(a)). Though the excitation provided by water flow and waves was much weaker than that provided by the vibration table in the experiment, significant difference still could be seen among the PSDTs depending on different transferring output DOFs (Figures 12(b) and 13(b)).

\section{Conclusions}

This paper presented the syntheticism of PSDT (4) and STF (18), which can be used to calculate the theoretical value of transmissibility with known transfer functions and excitations. In particular, because the theoretical power spectrum's amplitude of a random process is unrelated to frequency domain variables $(\omega)$, the amplitude of PSDT synthesis can be obtained by using (10) after appropriately choosing offdiagonal entries and establishing matrix $[\Gamma]$ according to the correlation of excitation. In addition, the amplitude of excitation can be replaced with its standard deviation to calculate STF syntheses by using (18) in the case of random excitation and to obtain better results. By comparison, it can be seen that the results obtained by the above calculation are 


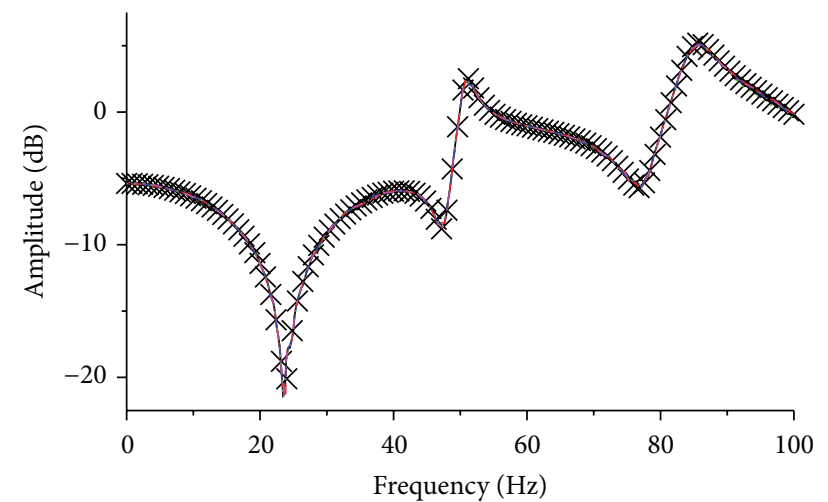

$\begin{array}{llll}\times & \text { STF synthesis } & \cdots . . & \text { PSDT measurement }(p=12) \\ & \text { PSDT measurement }(p=1) & \ldots- & \text { PSDT measurement }(p=17) \\ \ldots- & \text { PSDT measurement }(p=6) & \ldots- & \text { PSDT measurement }(p=19)\end{array}$

(a)

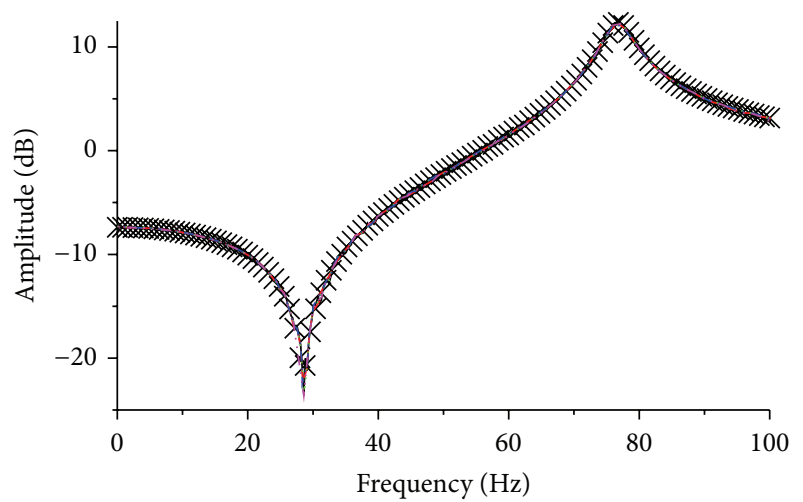

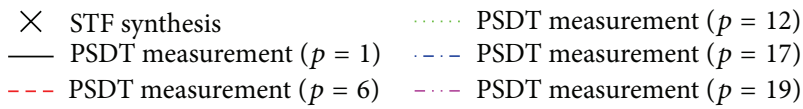

(b)

FIgURE 6: STF syntheses and PSDT measurements with point 4 as local DOF and point 17 as reference DOF in case 1 (a) and case 2 (b).

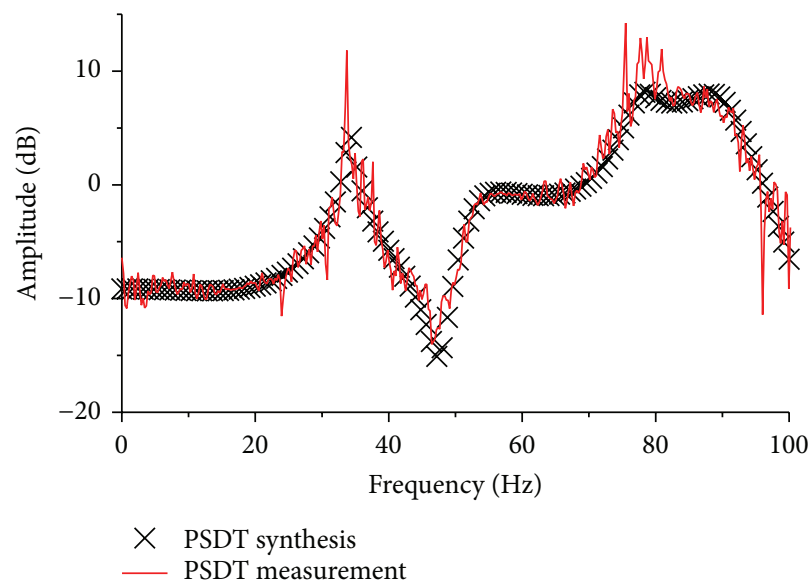

(a)

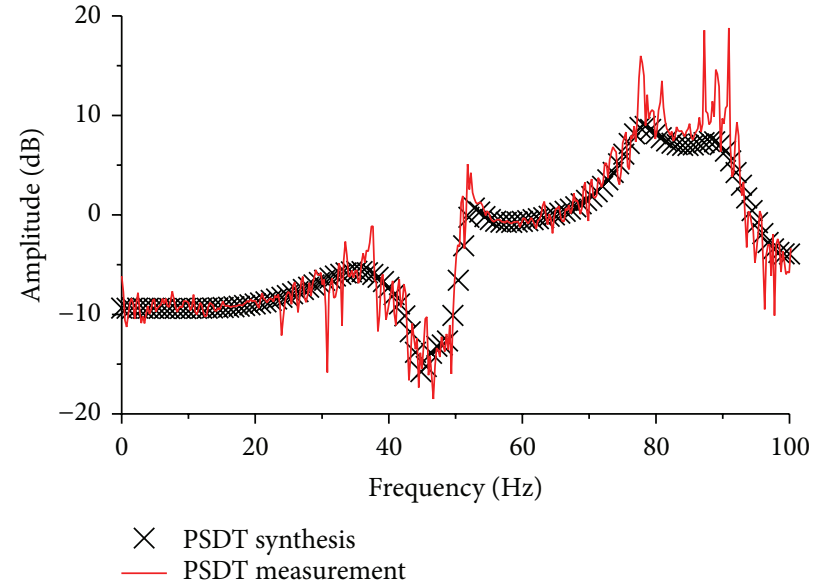

(b)

FIGURE 7: PSDT syntheses and measurements with point 4 as local DOF and point 17 as reference DOF in case 13 . (a) Choose point 5 as transferring output DOF; (b) choose point 15 as transferring output DOF.

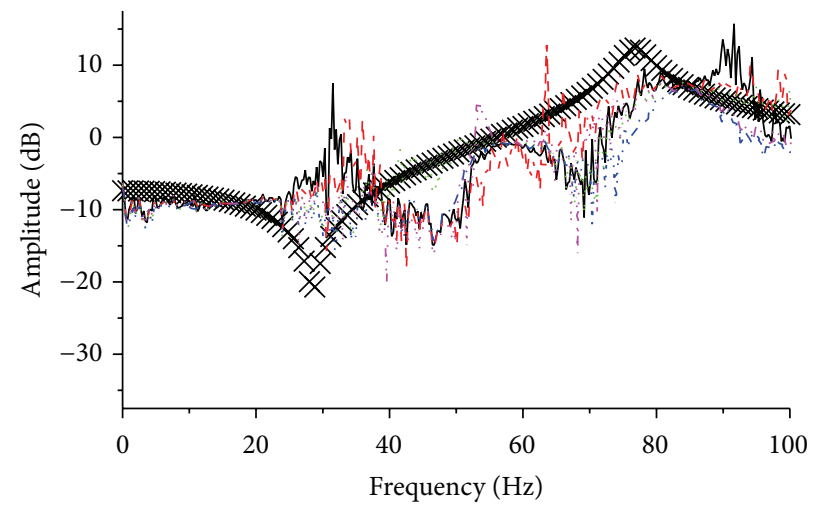

$$
\begin{aligned}
& \times \text { STF synthesis } \quad \cdots \ldots \text { PSDT measurement }(p=12) \\
& \text { — PSDT measurement }(p=1) \quad \ldots \text { PSDT measurement }(p=17) \\
& \text { - - - PSDT measurement }(p=6) \quad \ldots \text { PSDT measurement }(p=19)
\end{aligned}
$$

FigURE 8: PSDT measurements and STF synthesis in case 3 with point 4 as local DOF and point 17 as reference DOF. 


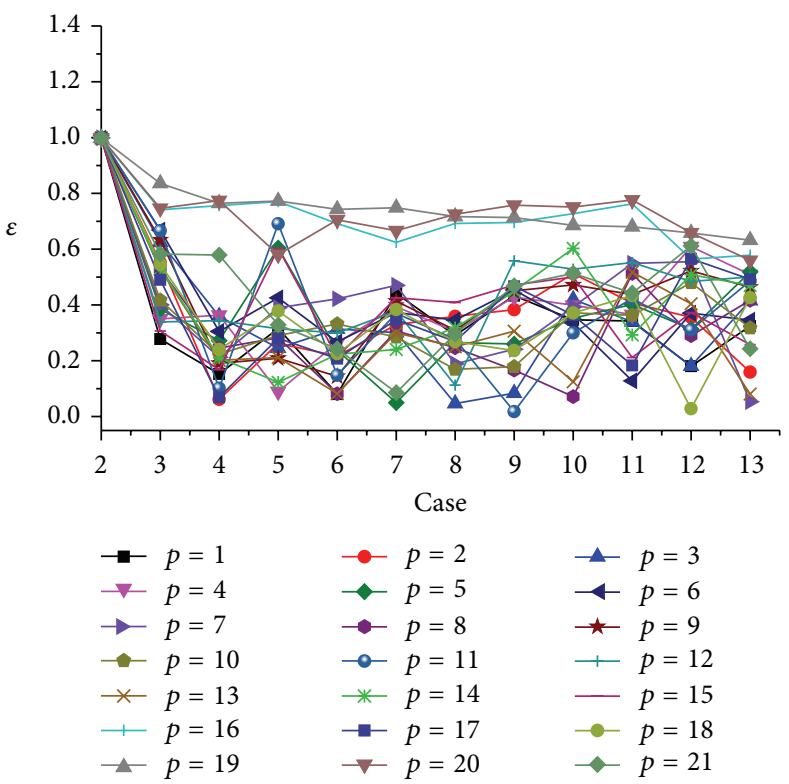

FIGURE 9: $\varepsilon$ in case 2-case 13 with point 4 as local DOF and point 17 as reference DOF.
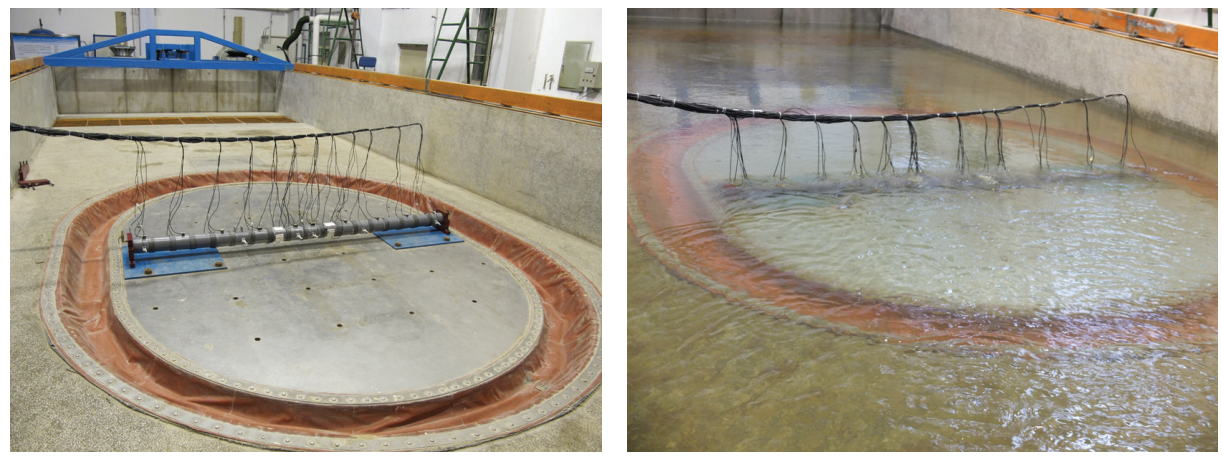

FIGURE 10: Submarine vibration-table test setup.

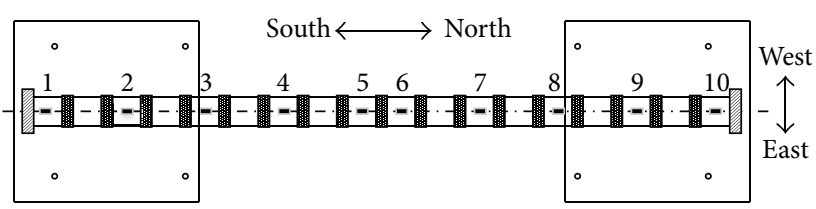

FIGURE 11: Measurement stations of underwater vibration-table test.

consistent with system response-based PSDT measurements obtained by using (1).

Based on the above syntheticism, this paper further explored some property of PSDT and reached the following conclusions:

(1) PSDT and STF are two physical quantities that are closely related to each other and different from each other. Under the loading condition of completely correlated excitations (including single excitation), the amplitude of PSDT, depending on same localreference DOF combination, coincides exactly with the amplitude of STF.
(2) If inputs are fully correlative to each other (including the situation of single input), PSDT's amplitude does not change with transferring output DOF $p$; or else, PSDT's amplitude changes with different modes of $p$ selection and is generally different from that of STF.

(3) In the case that excitations are not fully correlated, it seems that there is no obvious correlation between the similarity of PSDTs with the same local-reference DOF combination and the number of independent excitations.

The correctness of the above conclusions has been proven by the numerical experiment and underwater vibration-table model experiment.

\section{Conflict of Interests}

The authors declare that there is no conflict of interests regarding the publication of this paper. 


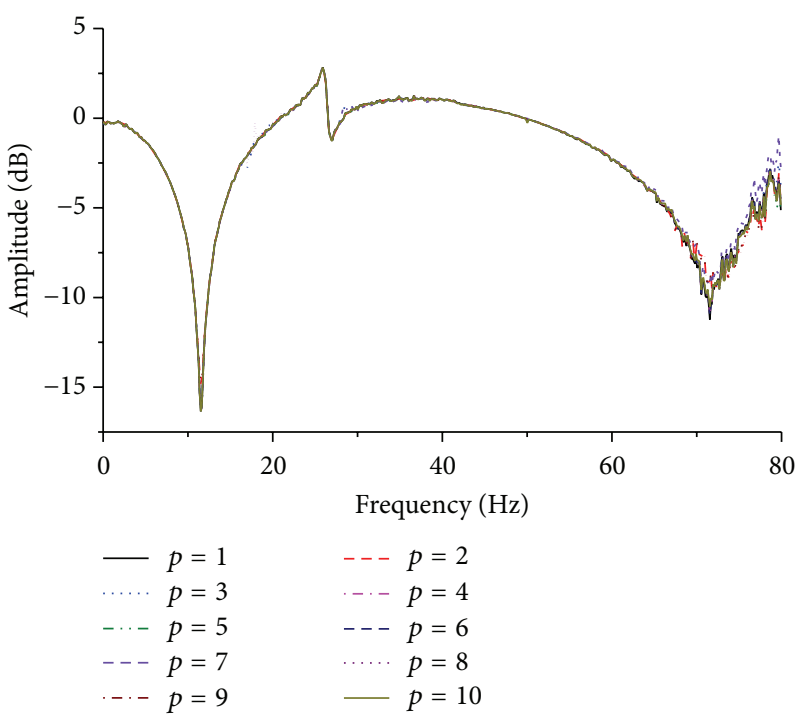

(a)

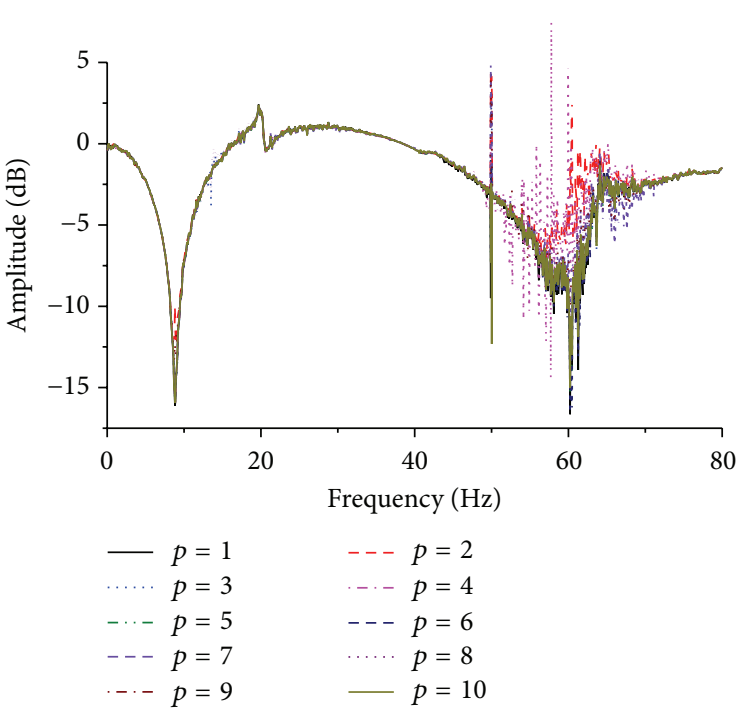

(b)

FIGURE 12: PSDT measurements with measurement stations 2 and 5 as local and reference station, respectively. (a) case 1; (b) case 2.

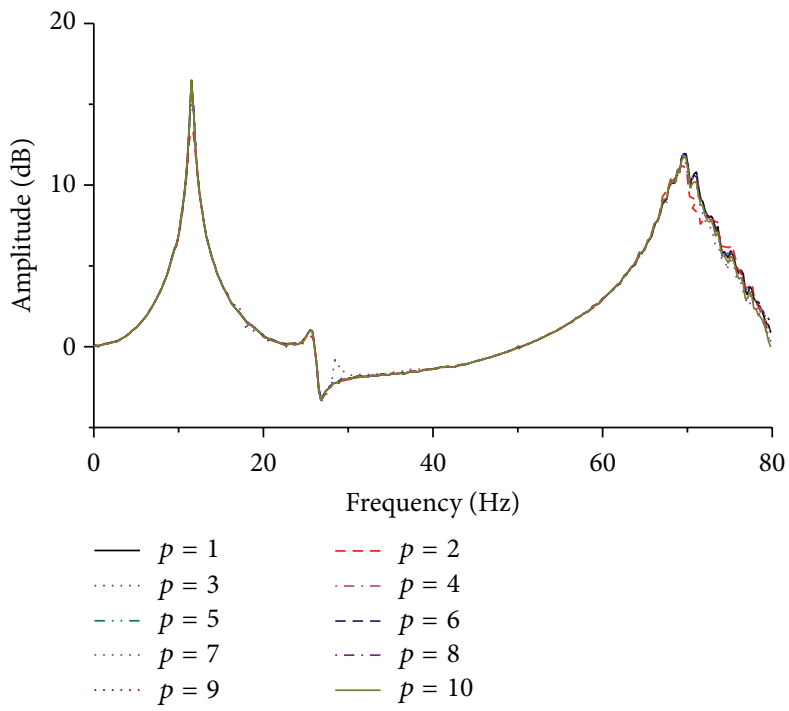

(a)

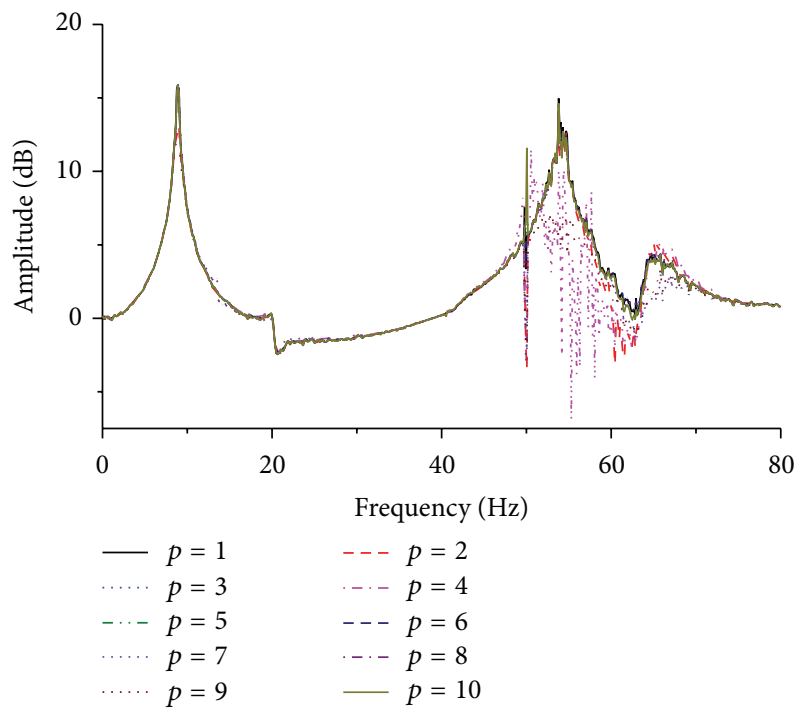

(b)

FIGURE 13: PSDT measurements with measurement stations 6 and 9 as local and reference station, respectively. (a) case 1; (b) case 2.

\section{Acknowledgment}

This study is supported by the Ministry of Science and Technology of China (Grants nos. 2011CB013702 and 2013CB035906) and National Natural Science Foundation of China (Grant no. 51378088).

\section{References}

[1] W.-J. Yan and W.-X. Ren, "Operational modal parameter identification from power spectrum density transmissibility," Computer-Aided Civil and Infrastructure Engineering, vol. 27, no. 3, pp. 202-217, 2012.
[2] C. Devriendt, P. Guillaume, and G. De Sitter, "Identification of modal parameters from transmissibility measurements with multiple loads," in Proceedings of the 12th International Congress on Sound and Vibration 2005 (ICSV '05), pp. 2919-2927, IEEE, Lisbon, Portugal, July 2005.

[3] C. Devriendt and P. Guillaume, "The use of transmissibility measurements in output-only modal analysis," Mechanical Systems and Signal Processing, vol. 21, no. 7, pp. 2689-2696, 2007.

[4] C. Devriendt and P. Guillaume, "Identification of modal parameters from transmissibility measurements," Journal of Sound and Vibration, vol. 314, no. 1-2, pp. 343-356, 2008.

[5] A. M. R. Ribeiro, J. M. M. Silva, and N. M. M. Maia, "On the generalisation of the transmissibility concept," Mechanical Systems and Signal Processing, vol. 14, no. 1, pp. 29-35, 2000. 
[6] M. Fontul, A. M. R. Ribeiro, J. M. M. Silva, and N. M. M. Maia, "Transmissibility matrix in harmonic and random processes," Shock and Vibration, vol. 11, no. 5-6, pp. 563-571, 2004.

[7] N. M. M. Maia, R. A. B. Almeida, and A. P. V. Urgueira, "Understanding transmissibility properties," in Proceedings of the Conference \& Exposition on Structural Dynamics-Technologies for Civil Structures (IMAC XXVI '08), Orlando, Fla, USA, February 2008.

[8] I. G. Araújo and J. E. Laier, "Operational modal analysis using SVD of power spectral density transmissibility matrices," Mechanical Systems and Signal Processing, vol. 46, no. 1, pp. 129145,2014

[9] J. S. Bendat and A. G. Piersol, Random Data: Analysis and Measurement Procedures, John Wiley \& Sons, 3rd edition, 2000.

[10] P. M. T. Broersen, "A comparison of transfer function estimators," IEEE Transactions on Instrumentation and Measurement, vol. 44, no. 3, pp. 657-661, 1995. 


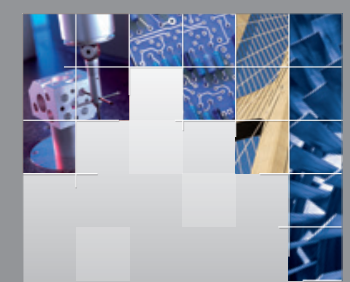

\section{Enfincering}
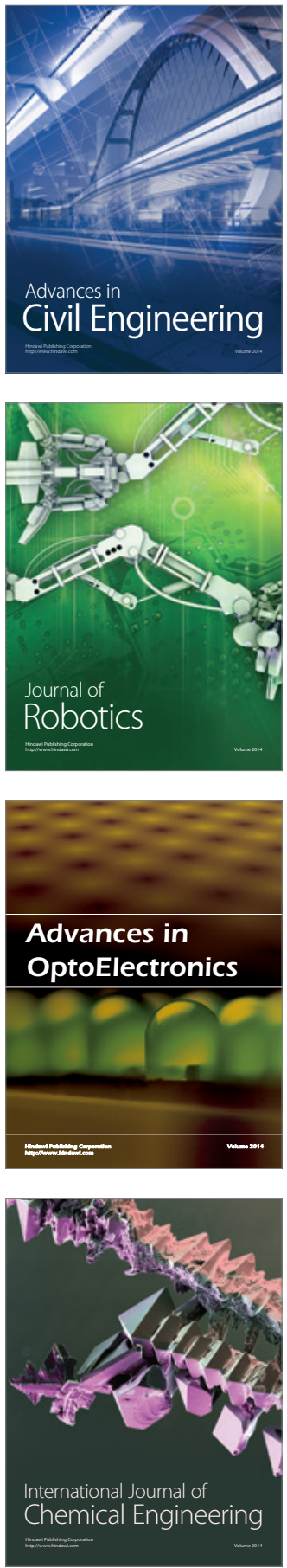

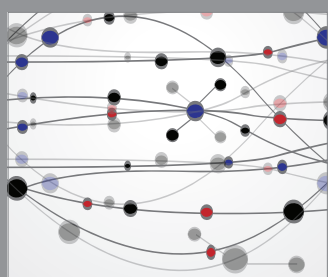

The Scientific World Journal

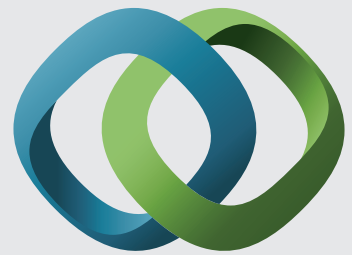

\section{Hindawi}

Submit your manuscripts at

http://www.hindawi.com
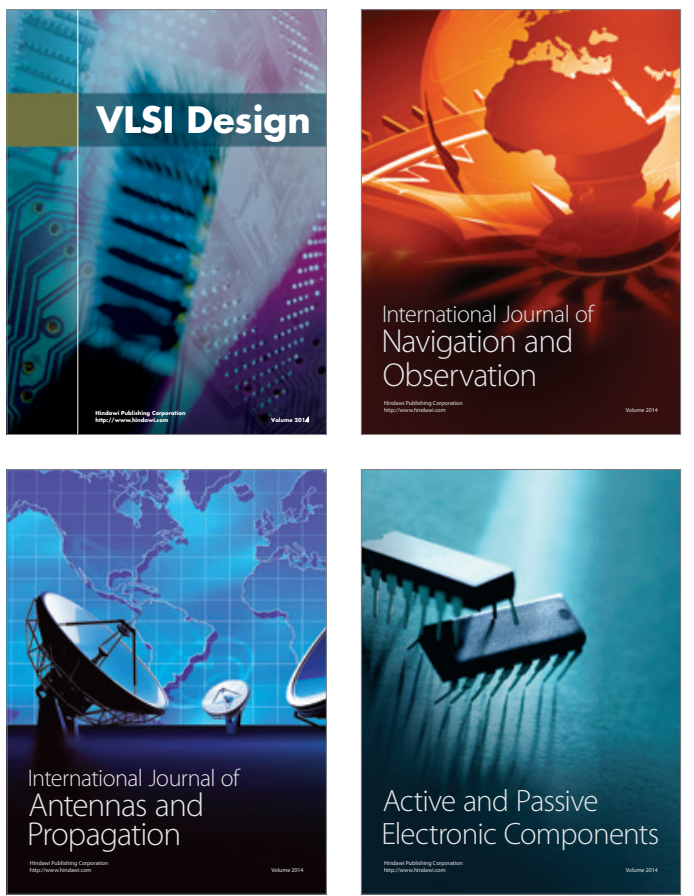
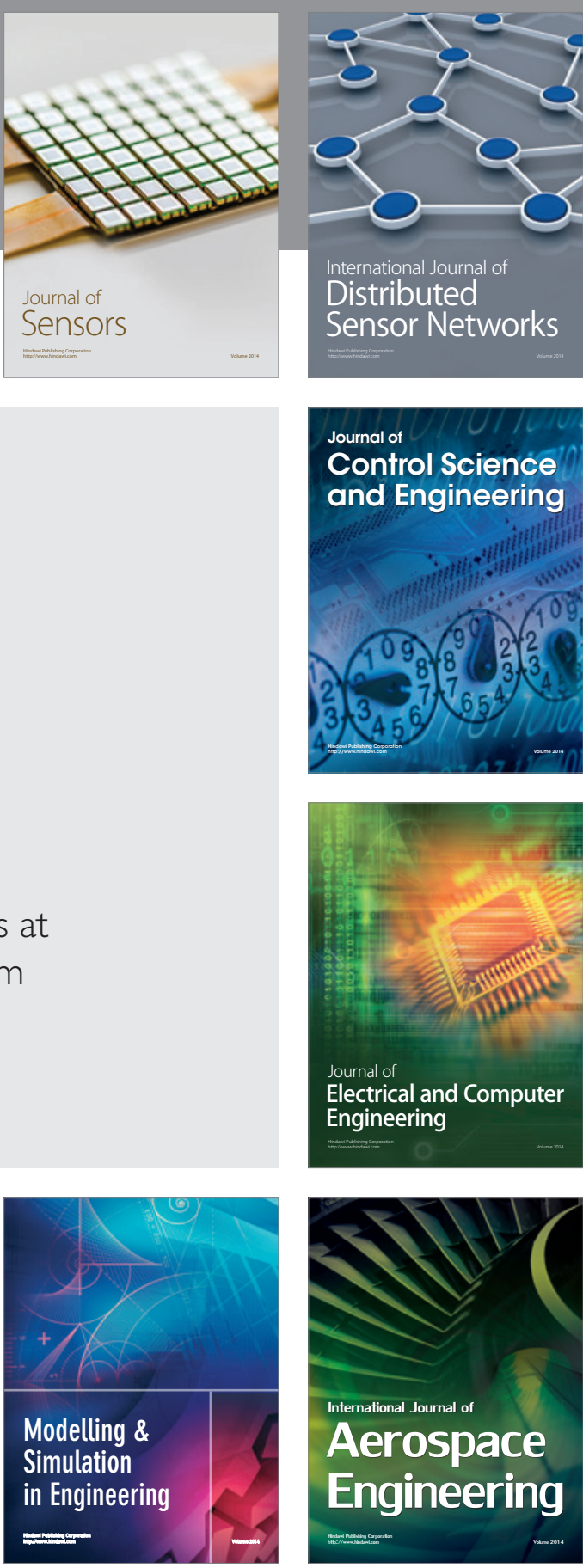

International Journal of

Distributed

Sensor Networks

Journal of

Control Science

and Engineering
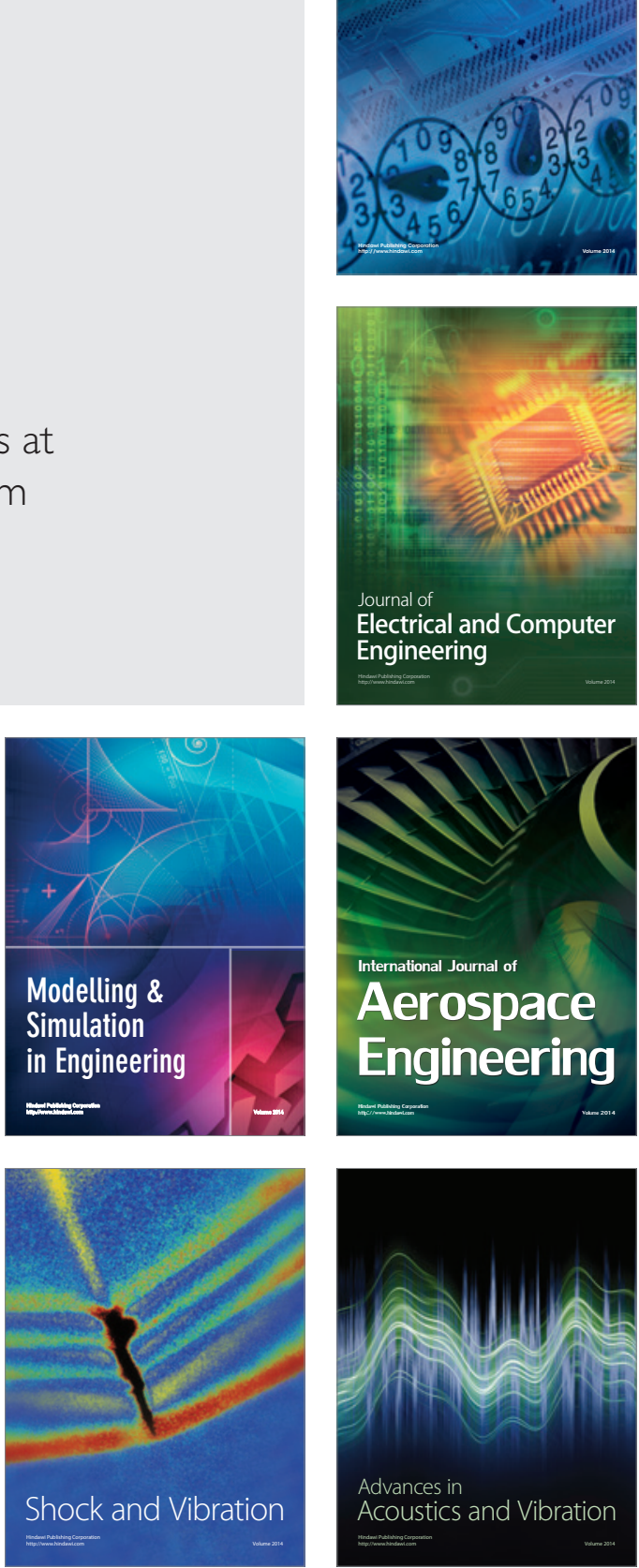